\title{
Mountain waves in two-layer sheared flows: critical-level effects, wave reflection, and drag enhancement
}

Article

Published Version

Teixeira, M. A.C., Miranda, P. M. A. and Argaín, J. (2008) Mountain waves in two-layer sheared flows: critical-level effects, wave reflection, and drag enhancement. Journal of the Atmospheric Sciences, 65 (6). pp. 1912-1926. ISSN 15200469 doi: https://doi.org/10.1175/2007JAS2577.1 Available at https://centaur.reading.ac.uk/29245/

It is advisable to refer to the publisher's version if you intend to cite from the work. See Guidance on citing.

Published version at: http://dx.doi.org/10.1175/2007JAS2577.1

To link to this article DOI: http://dx.doi.org/10.1175/2007JAS2577.1

Publisher: American Meteorological Society

All outputs in CentAUR are protected by Intellectual Property Rights law, including copyright law. Copyright and IPR is retained by the creators or other copyright holders. Terms and conditions for use of this material are defined in the End User Agreement.

www.reading.ac.uk/centaur 
Central Archive at the University of Reading

Reading's research outputs online 


\title{
Mountain Waves in Two-Layer Sheared Flows: Critical-Level Effects, Wave Reflection, and Drag Enhancement
}

\author{
Miguel A. C. Teixeira and Pedro M. A. Miranda \\ CGUL, IDL, University of Lisbon, Lisbon, Portugal \\ José L. Argaín \\ Department of Physics, University of Algarve, Faro, Portugal
}

(Manuscript received 31 July 2007, in final form 31 October 2007)

\begin{abstract}
Internal gravity waves generated in two-layer stratified shear flows over mountains are investigated here using linear theory and numerical simulations. The impact on the gravity wave drag of wind profiles with constant unidirectional or directional shear up to a certain height and zero shear above, with and without critical levels, is evaluated. This kind of wind profile, which is more realistic than the constant shear extending indefinitely assumed in many analytical studies, leads to important modifications in the drag behavior due to wave reflection at the shear discontinuity and wave filtering by critical levels. In inviscid, nonrotating, and hydrostatic conditions, linear theory predicts that the drag behaves asymmetrically for backward and forward shear flows. These differences primarily depend on the fraction of wavenumbers that pass through their critical level before they are reflected by the shear discontinuity. If this fraction is large, the drag variation is not too different from that predicted for an unbounded shear layer, while if it is small the differences are marked, with the drag being enhanced by a considerable factor at low Richardson numbers (Ri). The drag may be further enhanced by nonlinear processes, but its qualitative variation for relatively low $\mathrm{Ri}$ is essentially unchanged. However, nonlinear processes seem to interact constructively with shear, so that the drag for a noninfinite but relatively high $\mathrm{Ri}$ is considerably larger than the drag without any shear at all.
\end{abstract}

\section{Introduction}

Many studies on internal gravity waves, particularly those that are analytical, consider a background state where the velocity profile has a constant shear in the whole domain, or where the shear is piecewise constant. Examples are the seminal paper of Booker and Bretherton (1967) on critical levels; the studies of Klemp and Lilly (1975), Lindzen and Tung (1976), Miranda and Valente (1997), and Wang and Lin (1999), concerned with resonance, wave ducting, and downslope windstorms; Smith's $(1984,1986)$ theory of lee cyclogenesis; and Keller's (1994) study about nonhydrostatic effects. Other examples include the investigations on inertia-gravity waves of Shen and Lin (1999),

Corresponding author address: Miguel A. C. Teixeira, Centro de Geofisica da Universidade de Lisboa, Edificio C8, Campo Grande, 1749-016 Lisbon, Portugal.

E-mail: mateixeira@fc.ul.pt
Wurtele et al. (2000), Shutts (2001), and Öllers et al. (2003), or those on pure gravity waves by Shutts (1995), Grubišić and Smolarkiewicz (1997), Shutts and Gadian (1999), Sharman and Wurtele (2004), and Teixeira et al. (2005). This kind of wind profile is presumably used for mathematical convenience, because the solution to the linear gravity wave problem can then be expressed in terms of elementary functions when there is no rotation, and even if rotation is included the solution is still analytical. Among the studies concerned with mountain waves, the only ones where the impact of an infinite shear layer on the surface drag is evaluated are those of Smith (1986), Grubišić and Smolarkiewicz (1997), and Teixeira et al. (2005). In other studies (e.g., Shutts 1995; Shutts and Gadian 1999), the assumed Richardson number ( $\mathrm{Ri}$ ) is too large for the drag to be affected. It turns out that for a constant unidirectional shear spanning the whole atmosphere, the linear hydrostatic drag decreases with $\mathrm{Ri}$ in a way that does not depend on the sign of the shear, that is, whether the wind velocity 
increases or decreases with height (Grubišić and Smolarkiewicz 1997). On the other hand, at low Ri, discontinuities in the shear lead to resonances that modulate the drag with respect to the height where these discontinuities occur (Miranda and Valente 1997; Teixeira et al. 2005).

Now, a wind profile with constant shear that extends indefinitely is clearly unrealistic, and not easily implemented in numerical models. In such models, a wind that varies linearly near the surface must necessarily be bounded aloft, possibly with a transition to a constant value or to zero above some height (Shutts and Gadian 1999). If this transition is sufficiently slow, or occurs either inside a dissipation layer or above a critical level at sufficiently high $\mathrm{Ri}$, there is the possibility that downward wave reflections may be precluded. However, either for a forward unidirectional shear or when there is incomplete wave absorption by critical levels in a directionally sheared flow, a shear discontinuity is likely to have substantial effects on the surface drag. In numerical simulations where backward unidirectional shears have been used, it has been shown (Grubišić and Smolarkiewicz 1997) that the drag behavior follows exact linear predictions for an infinite shear layer quite closely, and is in even better agreement at low Ri with predictions obtained using a Wentzel-KramersBrillouin (WKB) approximation, for low mountain heights (Teixeira et al. 2004; Teixeira and Miranda 2004, 2006). While this behavior may be explained by critical-level absorption at high $\mathrm{Ri}$, it is not clear why it holds for $\mathrm{Ri}$ as small as $1 / 4$, where critical levels are in fact essentially transparent to gravity waves. The relatively small differences in drag behavior at low Ri that do exist in this case between numerical and analytical models have been attributed by Grubišić and Smolarkiewicz (1997) to nonlinear processes. In the present study, it is suggested that these differences may be due to wave reflection at a shear discontinuity existing above the critical level.

A finite shear layer, where the wind varies linearly from the surface up to a certain height and then becomes constant, is considered. For this flow, which can be viewed as the simplest possible realistic flow with shear, the variation of the surface gravity wave drag on $2 \mathrm{D}$ and $3 \mathrm{D}$ circular mountains is investigated. The model results, corroborated by numerical simulations, show that the drag behavior for positive and negative shears is highly asymmetric, unlike what is predicted by linear theory for an infinite shear layer.

This paper is organized as follows: Section 2 introduces the theoretical model of mountain waves in a two-layer atmosphere. Section 3 presents the results, beginning with a treatment of $2 \mathrm{D}$ unidirectional shear flow and proceeding with 3D directional shear. A comparison of the analytical results with numerical simulations and an evaluation of nonlinear effects are carried out next. Finally, section 4 contains the concluding remarks.

\section{Internal gravity waves in a two-layer atmosphere}

As will be seen later in this paper, the effects of a two-layer atmosphere on the surface gravity wave drag depend essentially on whether the gravity waves are filtered by passage through a critical level before they are reflected by a shear discontinuity or not. Thus, the drag behavior is fundamentally different for unidirectional or directional shear flows. In the first case, all wavenumbers either have or do not have a single critical level (where the wind speed is zero) before they are reflected, while in the second case some wavenumbers pass through a critical level (where the wavenumber and wind vectors are perpendicular) before they are reflected, and others do not.

The behavior of unidirectional shear flows is particularly easy to study in the case of flow over 2D mountains. Understanding the behavior of directionally sheared flows, on the other hand, requires using 3D orography. For this reason, flow over a $2 \mathrm{D}$ ridge and a circular mountain will be considered. When the inviscid, nonrotating, hydrostatic, linearized equations of motion with the Boussinesq approximation are combined, a single equation for the vertical velocity perturbation results. The Fourier transform of the vertical velocity perturbation $\hat{w}$ satisfies

$$
\hat{w}^{\prime \prime}+\frac{N^{2}\left(k^{2}+l^{2}\right)}{(U k+V l)^{2}} \hat{w}=0
$$

where $(U, V)$ is the undisturbed wind velocity, $(k, l)$ is the horizontal wavenumber, $N$ is the Brunt-Väisälä frequency of the incoming flow, and the primes denote differentiation with respect to height $z$. In (1), it has been noted that only piecewise linear wind profiles will be used, so the curvature terms do not appear. This equation is singular at critical levels, where $U k+V l=0$.

An undisturbed wind profile that is linear up to a certain height $z_{1}$ and then becomes constant, but that does not have any other constraint, is assumed, that is,

$$
\begin{aligned}
& U=U_{0}+\alpha z \text { and } V=V_{0}+\beta z, \quad \text { if } z<z_{1}, \quad \text { or } \\
& U=U_{0}+\alpha z_{1}=U_{1} \text { and } V=V_{0}+\beta z_{1}=V_{1}, \quad \text { if } \\
& z>z_{1},
\end{aligned}
$$


where $U_{0}, V_{0}, \alpha, \beta, U_{1}$, and $V_{1}$ are constants. This represents a rather general class of flows. For this type of wind profile, the solution to (1) in the layer $z<z_{1}$ is of the form

$$
\begin{aligned}
\hat{w}= & \gamma\left(1+\frac{\alpha k+\beta l}{U_{0} k+V_{0} l} z\right)^{1 / 2-i \mu s} \\
& +\delta\left(1+\frac{\alpha k+\beta l}{U_{0} k+V_{0} l} z\right)^{1 / 2+i \mu s},
\end{aligned}
$$

corresponding to both an upward- and a downwardpropagating wave, where $\gamma(k, l)$ and $\delta(k, l)$ are functions to be determined, $s=\operatorname{sign}(\alpha k+\beta l)$, and

$$
\mu=\left[\frac{N^{2}\left(k^{2}+l^{2}\right)}{(\alpha k+\beta l)^{2}}-\frac{1}{4}\right]^{1 / 2} .
$$

On the other hand, in the upper layer, the solution to (1) takes the form

$$
\hat{w}=\varepsilon \exp \left[i \frac{N\left(k^{2}+l^{2}\right)^{1 / 2}}{U_{1} k+V_{1} l} z\right]
$$

where $\varepsilon(k, l)$ is a function to be determined.

The boundary conditions that must be applied to (1) are the following: the flow must be tangential to the orography at the surface, namely,

$$
\hat{w}(z=0)=i\left(U_{0} k+V_{0} l\right) \hat{h},
$$

where $\hat{h}$ is the Fourier transform of the terrain elevation (assumed to correspond to an isolated mountain), and the wave energy must radiate upward in the upper layer. This last condition is already included in the solution (5). Additionally, both $\hat{w}$ and the pressure perturbation (or rather its Fourier transform $\hat{p}$ ) must be continuous at $z=z_{1}$. From the equations of motion, the Fourier transform of the pressure perturbation may be related to $\hat{w}$ and its vertical derivative through

$$
\hat{p}=i \frac{\rho_{0}}{k^{2}+l^{2}}\left[\left(U^{\prime} k+V^{\prime} l\right) \hat{w}-(U k+V l) \hat{w}^{\prime}\right],
$$

where $\rho_{0}$ is a reference density (assumed to be constant). If (3) and (5) are used in conjunction with (7), and (6) is also applied, then it is possible to determine the unknown functions $\gamma, \delta$, and $\varepsilon$. For the purpose of calculating the surface drag, it is only necessary to know the explicit form of $\gamma$ and $\delta$, which can be shown to be the following:

$$
\begin{gathered}
\gamma=\frac{i\left(U_{0} k+V_{0} l\right) \hat{h}\left[i \mu s-\frac{1}{2}-i \frac{N\left(k^{2}+l^{2}\right)^{1 / 2}}{\alpha k+\beta l}\right]\left(\frac{U_{1} k+V_{1} l}{U_{0} k+V_{0} l}\right)^{i \mu s}}{\left[i \mu s-\frac{1}{2}-i \frac{N\left(k^{2}+l^{2}\right)^{1 / 2}}{\alpha k+\beta l}\right]\left(\frac{U_{1} k+V_{1} l}{U_{0} k+V_{0} l}\right)^{i \mu s}+\left[i \mu s+\frac{1}{2}+i \frac{N\left(k^{2}+l^{2}\right)^{1 / 2}}{\alpha k+\beta l}\right]\left(\frac{U_{1} k+V_{1} l}{U_{0} k+V_{0} l}\right)^{-i \mu s}}, \quad \text { and } \\
\delta=\frac{i\left(U_{0} k+V_{0} l\right) \hat{h}\left[i \mu s+\frac{1}{2}+i \frac{N\left(k^{2}+l^{2}\right)^{1 / 2}}{\alpha k+\beta l}\right]\left(\frac{U_{1} k+V_{1} l}{U_{0} k+V_{0} l}\right)^{-i \mu s}}{\left[i \mu s-\frac{1}{2}-i \frac{N\left(k^{2}+l^{2}\right)^{1 / 2}}{\alpha k+\beta l}\right]\left(\frac{U_{1} k+V_{1} l}{U_{0} k+V_{0} l}\right)^{i \mu s}+\left[i \mu s+\frac{1}{2}+i \frac{N\left(k^{2}+l^{2}\right)^{1 / 2}}{\alpha k+\beta l}\right]\left(\frac{U_{1} k+V_{1} l}{U_{0} k+V_{0} l}\right)^{-i \mu s}} .
\end{gathered}
$$

The remaining coefficient $\varepsilon$ may be calculated in terms of $\gamma$ and $\delta$. This totally determines the solution to the gravity wave problem in a generic $3 \mathrm{D}$ case. To obtain the solutions for the $2 \mathrm{D}$ case, it suffices to assume $l=0, V_{0}=$ $0, \beta=0$, and $V_{1}=0$ (with the understanding that the Fourier transforms denoted by a hat are now $1 \mathrm{D}$ and integrated over $x$, instead of 2D and integrated over $x$ and $y$ ).

\section{Mountain wave drag}

The drag force exerted by the flow on the orography is given in general by

$$
\left(D_{x}, D_{y}\right)=4 \pi^{2} i \int_{-\infty}^{+\infty} \int_{-\infty}^{+\infty}(k, l) \hat{p}^{*}(z=0) \hat{h} d k d l
$$

in a 3D situation, while the drag per unit length on a $2 \mathrm{D}$ ridge is given by

$$
D=2 \pi i \int_{-\infty}^{+\infty} k \hat{p}^{*}(z=0) \hat{h} d k
$$

where the asterisk denotes the complex conjugate (see Teixeira et al. 2004; Teixeira and Miranda 2004). These two drags differ in nature as they stand, but when normalized by the corresponding values for a constant wind equal to the wind at the surface $\left[\left(U_{0}, V_{0}\right)\right.$ or $U_{0}$, respectively], they become comparable (see Teixeira et al. 2005).

The Fourier transform of the pressure perturbation at the surface, which is necessary in (9) or (10), may be expressed in terms of $\gamma$ and $\delta$, presented in the previous subsection, as 


$$
\begin{aligned}
& \hat{p}(z=0) \\
& \quad=i \frac{\rho_{0}}{k^{2}+l^{2}}(\alpha k+\beta l)\left[\left(\frac{1}{2}+i \mu s\right) \gamma+\left(\frac{1}{2}-i \mu s\right) \delta\right] .
\end{aligned}
$$

Obviously, the drag may be obtained by taking the complex conjugate of (11), using the definitions of $\gamma$ and $\delta[(8)]$, and inserting them into (9) or (10) after appropriate simplification.

The actual final explicit form of the gravity wave drag depends on whether the factor $\left(U_{1} k+V_{1} l\right) /\left(U_{0} k+V_{0} l\right)$ contained in (8) is positive or negative. If it is positive, this means that upward-propagating gravity waves with the horizontal wavenumber $(k, l)$ have not passed through their specific critical level, where $U k+V l=0$, before they reach $z=z_{1}$, and so (a)
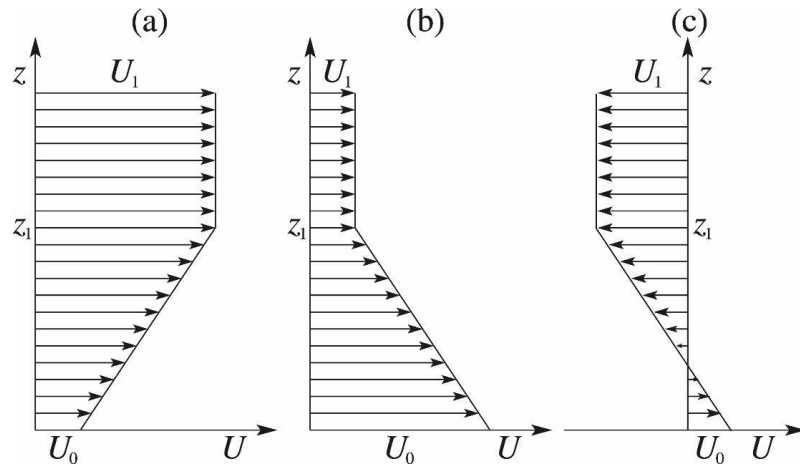

FIG. 1. Schematic diagram of the wind profiles with unidirectional shear considered in this study: (a) forward shear, (b) backward shear without a critical level, and (c) backward shear with a critical level.

$$
\begin{aligned}
\left(\frac{U_{1} k+V_{1} l}{U_{0} k+V_{0} l}\right)^{i \mu s} & =\cos \left(\mu s \log \left|\frac{U_{1} k+V_{1} l}{U_{0} k+V_{0} l}\right|\right)+i \sin \left(\mu s \log \left|\frac{U_{1} k+V_{1} l}{U_{0} k+V_{0} l}\right|\right), \quad \text { and }\left(\frac{U_{1} k+V_{1} l}{U_{0} k+V_{0} l}\right)^{-i \mu s} \\
& =\cos \left(\mu s \log \left|\frac{U_{1} k+V_{1} l}{U_{0} k+V_{0} l}\right|\right)-i \sin \left(\mu s \log \left|\frac{U_{1} k+V_{1} l}{U_{0} k+V_{0} l}\right|\right) .
\end{aligned}
$$

If, on the other hand, $\left(U_{1} k+V_{1} l\right) /\left(U_{0} k+V_{0} l\right)<0$, this means that the waves passed through a critical level before reaching $z=z_{1}$. Then it may be noted that because

$$
\frac{U_{1} k+V_{1} l}{U_{0} k+V_{0} l}=\left|\frac{U_{1} k+V_{1} l}{U_{0} k+V_{0} l}\right| e^{i \pi s}
$$

then

$$
\begin{gathered}
\left(\frac{U_{1} k+V_{1} l}{U_{0} k+V_{0} l}\right)^{i \mu s}=e^{-\pi \mu}\left[\cos \left(\mu s \log \left|\frac{U_{1} k+V_{1} l}{U_{0} k+V_{0} l}\right|\right)+i \sin \left(\mu s \log \left|\frac{U_{1} k+V_{1} l}{U_{0} k+V_{0} l}\right|\right)\right], \\
\left(\frac{U_{1} k+V_{1} l}{U_{0} k+V_{0} l}\right)^{-i \mu s}=e^{\pi \mu}\left[\cos \left(\mu s \log \left|\frac{U_{1} k+V_{1} l}{U_{0} k+V_{0} l}\right|\right)-i \sin \left(\mu s \log \left|\frac{U_{1} k+V_{1} l}{U_{0} k+V_{0} l}\right|\right)\right] .
\end{gathered}
$$

All of these equations are consistent with the idea, dictated by causality, that an upward-propagating wave is attenuated above the critical level, while a downwardpropagating wave is attenuated below the same critical level. Whether (12), (14), or both formulas have to be used in the drag integrals depends on the type of flow being considered. We will begin by treating $2 \mathrm{D}$ unidirectional shear flows, where critical levels, if they do exist, affect the whole spectrum of wavenumbers, and where the drag in fact has a closed analytical form. We will then proceed to 3D flows with directional shear, where only some wavenumbers have a critical level and the drag must be calculated numerically, albeit through the evaluation of a simple 1D integral (cf. Teixeira et al. 2005).

\section{Results}

\section{a. Unidirectional shear flow over a $2 D$ ridge}

\section{1) No Critical LeVel}

Consider a mountain ridge aligned in the $y$ direction and an incoming flow given by (2), where $V_{0}=\beta=$ $V_{1}=0, U_{0}>0$, and $\alpha>0$, that is, a unidirectional flow with forward shear along the $x$ direction (see Fig. 1a). In this case, the internal waves have no critical levels. Although we have assumed that $\alpha>0$, in fact the same theoretical framework can be applied to a backward shear flow that does not have a critical level (Fig. 1b). In both of these cases, (12) should be used in the drag 
calculations, and after some algebra the final obtained drag expression is

$$
\begin{aligned}
& D=4 \pi \rho_{0} N U_{0} \int_{0}^{+\infty} k|\hat{h}|^{2} d k \\
& \times \frac{\mu^{2} / \mathrm{Ri}}{1-\frac{1}{4 \mathrm{Ri}} \cos \left(2 \mu \log \left|\frac{U_{1}}{U_{0}}\right|\right)-\frac{\mu}{2 \mathrm{Ri}} \sin \left(2 \mu \log \left|\frac{U_{1}}{U_{0}}\right|\right)},
\end{aligned}
$$

where $\mathrm{Ri}=N^{2} / \alpha^{2}$. It is easy to note that, because of the hydrostatic approximation, the drag expression could be separated into an integral involving $\hat{h}$ and a fraction containing all the effects of shear (cf. Teixeira and Miranda 2004, 2005). This enables the drag to be normalized by its value for a constant wind equal to $U_{0}$,

$$
D_{0}=4 \pi \rho_{0} N U_{0} \int_{0}^{+\infty} k|\hat{h}|^{2} d k,
$$

yielding the normalized drag

$$
\begin{aligned}
& \frac{D}{D_{0}} \\
& =\frac{\mu^{2} / \mathrm{Ri}}{1-\frac{1}{4 \mathrm{Ri}} \cos \left(2 \mu \log \left|\frac{U_{1}}{U_{0}}\right|\right)-\frac{\mu}{2 \mathrm{Ri}} \sin \left(2 \mu \log \left|\frac{U_{1}}{U_{0}}\right|\right)} .
\end{aligned}
$$

This dimensionless drag is independent of the detailed shape of the orography as long as this is $2 \mathrm{D}$, and depends on the following two dimensionless parameters: $\mathrm{Ri}$ [contained also in $\mu$, which now becomes $\mu=(\mathrm{Ri}-$ $\left.1 / 4)^{1 / 2}\right]$ and $\left|U_{1} / U_{0}\right|$. This last parameter is linearly related to the lower-layer height $z_{1}$ through $U_{1} / U_{0}=1+$ $\alpha z_{1} / U_{0}$.

The drag for a shear layer that extends indefinitely is given by exact linear theory (see Smith 1986) as

$$
\frac{D}{D_{0}}=\frac{\mu}{\mathrm{Ri}^{1 / 2}}=\left(1-\frac{1}{4 \mathrm{Ri}}\right)^{1 / 2}
$$

while the drag predicted by a linear model employing the WKB approximation (Teixeira and Miranda 2004) is

$$
\frac{D}{D_{0}}=1-\frac{1}{8 \mathrm{Ri}} \text {. }
$$

Obviously, the exact expression (18), valid for arbitrarily large shears (as long as $\mathrm{Ri}>1 / 4$ ), tends asymptotically to (19) for high Ri. Note also that neither (18) nor (19) depend on the sign of $\alpha$.

It can be seen that the drag for a limited shear layer (17) oscillates with $\left|U_{1} / U_{0}\right|$, clearly due to wave reflec-
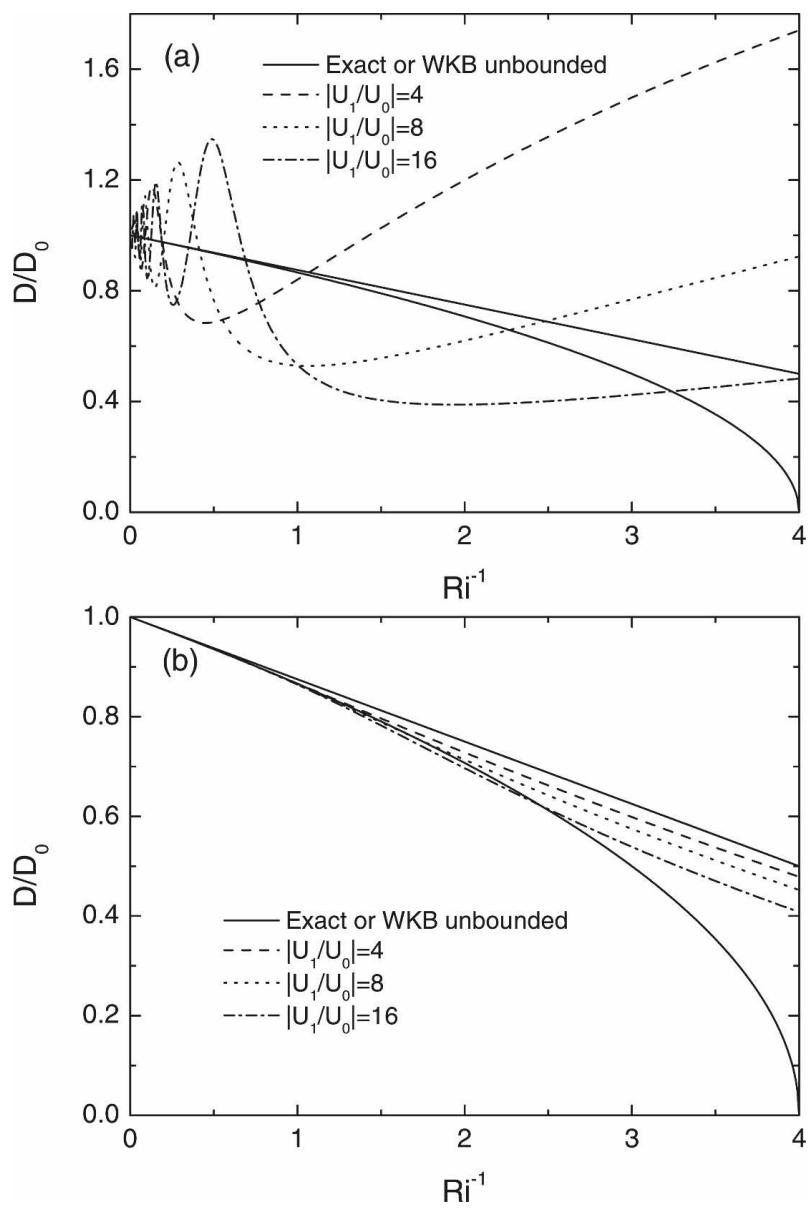

FIG. 2. Normalized drag as a function of $\mathrm{Ri}^{-1}$ for unidirectional shear flow over a 2D ridge: (a) no critical level, (b) one critical level.

tions at $z=z_{1}$ and constructive or destructive interference between upward- and downward-propagating waves in the layer $z<z_{1}$ [much in the same way as in Teixeira et al. (2005)]. Figure 2 a shows the variation of the normalized drag, as given by (19) and (18) (upper and lower solid lines, respectively), together with the normalized drag given by (17) for three values of $\left|U_{1}\right|$ $U_{0} \mid$ (dashed, dotted, and dash-dotted lines). It can be seen that, apart from the fact that the drag equals one at $\mathrm{Ri}^{-1}=0$, the drag curves for a finite shear layer show no resemblance to those that assume a shear that extends indefinitely. The drag for a finite shear layer oscillates around the solid curves with a rapidly decreasing period but increasing amplitude as $\mathrm{Ri}^{-1}$ goes from 0 to 4 . In this way, the drag takes values larger than 1.5 for high $\mathrm{Ri}^{-1}$, which are not predicted by the theories of Smith (1986) or Teixeira and Miranda (2004). No matter what finite value of $\left|U_{1} / U_{0}\right|$ is used, the drag oscillation never disappears, because in (17) the logarithm of $\left|U_{1} / U_{0}\right|$ is taken. Hence, either very small or very 
large values of $\left|U_{1} / U_{0}\right|$ lead to very large absolute values of this logarithm, which cause rapid oscillations of the denominator in (17). Physically, this behavior means that, no matter how high the shear discontinuity is, it will always reflect the waves (there is no wave dispersion in 2D flow). Thus, this case is intrinsically different from that of an unlimited shear layer. Only in the limits $\mathrm{Ri}^{-1}=0$ or $\left|U_{1} / U_{0}\right|=1$ do the drag oscillations disappear, and the drag equals one, but these cases are of no interest, because they correspond to a vanishing shear layer.

The limit of the drag as $\mathrm{Ri} \rightarrow 1 / 4$ may be shown to be

$$
\frac{D(\mathrm{Ri}=1 / 4)}{D_{0}}=\frac{1}{1-\log \left|\frac{U_{1}}{U_{0}}\right|+\frac{1}{2} \log ^{2}\left|\frac{U_{1}}{U_{0}}\right|} .
$$

This expression is plotted in Fig. 3a, together with the normalized drag for other values of $\mathrm{Ri}^{-1}$, as a function of $\left|U_{1} / U_{0}\right|$. It can be shown that the drag at $\mathrm{Ri}=1 / 4$ attains a maximum of 2 at $\left|U_{1} / U_{0}\right|=e \approx 2.7$. As anticipated by the fact that logarithms are taken in (20), the drag behavior is essentially symmetric with respect to its maximum (in a logarithmic scale), tending to zero both for very high and very low $\left|U_{1} / U_{0}\right|$. On the other hand, for higher values of $\mathrm{Ri}$, the drag is periodic with $\log \left|U_{1} / U_{0}\right|$, having a modulation with both amplitude and period that increase as $\mathrm{Ri}$ decreases, as suggested by Fig. 2a. This is due to the factors of $\mu$ inside the sine and cosine functions in the denominator of (17), and to the fact that the coefficients multiplying these functions become larger as Ri decreases.

\section{2) One CRitical Level}

Consider now an incoming flow given by (2), where $V_{0}=\beta=V_{1}=0, U_{0}>0, \alpha<0$, and $U_{1}<0$, that is, a unidirectional flow with backward shear and a critical level (see Fig. 1c). In this case, a fraction of the wave energy passes through the critical level twice-first as it
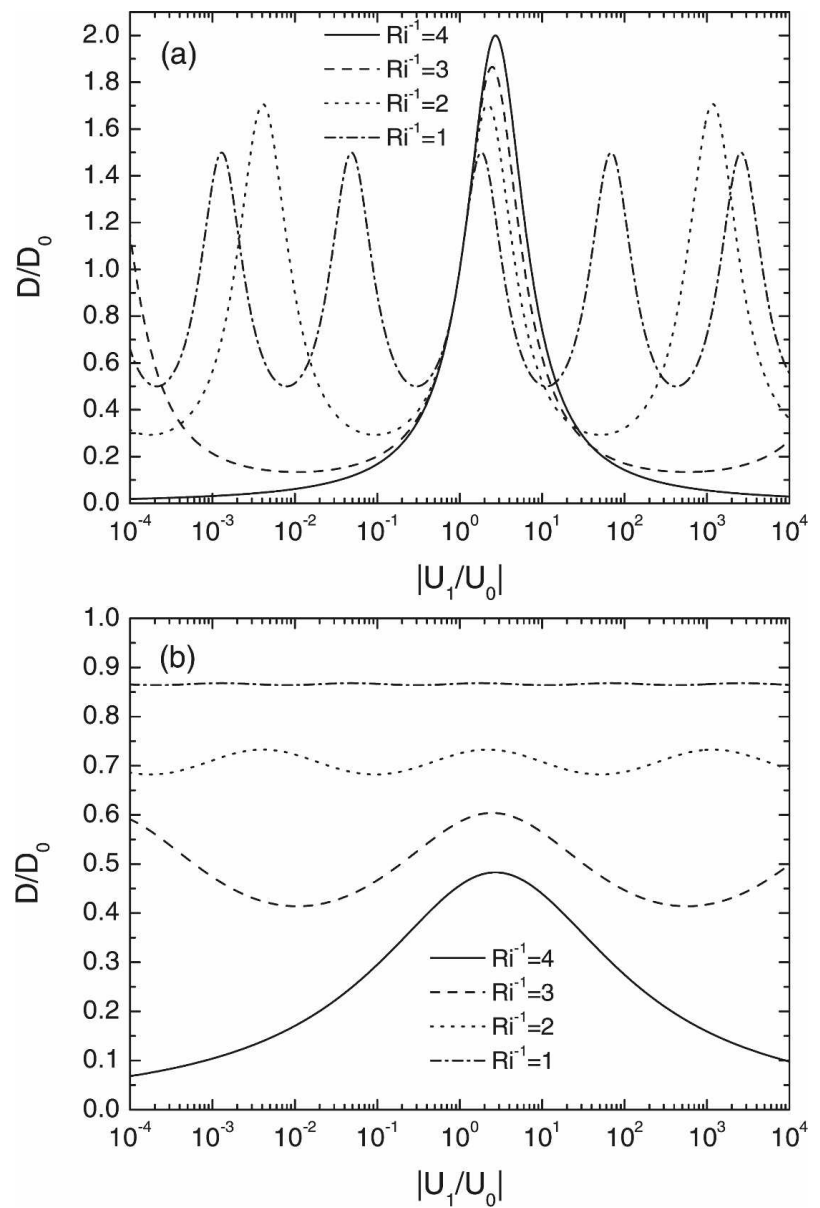

FIG. 3. Normalized drag at as a function of $\left|U_{1} / U_{0}\right|$ for unidirectional shear flow over a 2D ridge: (a) no critical level, (b) one critical level.

propagates upward and then as it is reflected back toward the surface. Then, (14) should be used for all wavenumbers in the calculation of the drag, and the final expression for the normalized drag may be shown to be

$$
\frac{D}{D_{0}}=\frac{\frac{\mu^{2}}{\mathrm{Ri}} \cosh (2 \pi \mu)+\frac{\mu}{\mathrm{Ri}^{1 / 2}} \sinh (2 \pi \mu)}{\cosh (2 \pi \mu)+\frac{\mu}{\mathrm{Ri}^{1 / 2}} \sinh (2 \pi \mu)-\frac{1}{4 \mathrm{Ri}} \cos \left(2 \mu \log \left|\frac{U_{1}}{U_{0}}\right|\right)-\frac{\mu}{2 \mathrm{Ri}} \sin \left(2 \mu \log \left|\frac{U_{1}}{U_{0}}\right|\right)},
$$

where the terms involving $2 \pi \mu$ result from (14) and ultimately from the branch line of the logarithmic function. Physically, these terms result from filtering of the gravity waves by the critical level, and considerably modify the behavior of the drag, as will be seen next.

Figure $2 \mathrm{~b}$ shows the normalized drag as a function of $\mathrm{Ri}^{-1}$ as given by (21) (dashed, dotted, and dash-dotted line), and by (18) and (19) (solid lines). It can be seen that the drag has a much more regular behavior than in the case of no critical level. For the selected values of $\left|U_{1} / U_{0}\right|$, the drag follows the exact and WKB results at relatively high Ri very closely, while it falls between the two corresponding curves, and surprisingly stays closer to the WKB result, for lower $\mathrm{Ri}$. It can be shown that, 
unlike (17), (21) tends asymptotically to (18) for $\mathrm{Ri} \rightarrow$ $+\infty$. The reason for this is very simple: for high values of $\mathrm{Ri}$, the upward-propagating waves are essentially absorbed by the critical level [they are in fact attenuated by a factor of $\exp (-\pi \mu)$, as shown by Booker and Bretherton (1967)], so the waves do not "feel" what is above this level, and no downward-propagating wave exists in the layer $z<z_{1}$.

In the limit $\mathrm{Ri} \rightarrow 1 / 4$, (21) reduces to

$$
\frac{D(\mathrm{Ri}=1 / 4)}{D_{0}}=\frac{1+\pi}{\frac{\pi^{2}}{2}+\pi+1-\log \left|\frac{U_{1}}{U_{0}}\right|+\frac{1}{2} \log ^{2}\left|\frac{U_{1}}{U_{0}}\right|}
$$

It may be shown that this function has a maximum of $(1+\pi) /\left(\pi^{2} / 2+\pi+1 / 2\right) \approx 0.48$ at $\left|U_{1} / U_{0}\right|=e \approx 2.7$. It is fortuitous that this value is so close to the value of 0.5 predicted for the normalized drag by the model using the WKB approximation (19) at the same Ri.

A more careful analysis of what happens at low Ri may be aided by Fig. 3b, where the drag is plotted as a function of $\left|U_{1} / U_{0}\right|$ for various $\mathrm{Ri}$, including $\mathrm{Ri}=1 / 4$. As in the case of no critical level, the period and amplitude of the drag oscillations increases as $\mathrm{Ri}$ decreases, but the amplitude, particularly for $\mathrm{Ri} \neq 1 / 4$, is much smaller than in the previous case. This is consistent with the fact that the drag oscillates much less with $\mathrm{Ri}$ in Fig. 2b than in Fig. 2a.

Using (8), it is not hard to show that the reflection coefficient of the flow under consideration is

$$
R=\left|\frac{\gamma}{\delta}\right|=\left(\frac{1-\mu / \mathrm{Ri}^{1 / 2}}{1+\mu / \mathrm{Ri}^{1 / 2}}\right)^{1 / 2} e^{-2 \pi \mu}
$$

[a similar definition, without the exponential factor on the right, is valid in the case of forward shear, as noted by Keller (1994)]. The exponential factor, corresponding to double absorption at the critical level (of upwardand downward-propagating waves), means that if $\mathrm{Ri}$ is substantially above $1 / 4$ the reflection coefficient is very low, because the waves are unable to reach the shear discontinuity, where they are reflected back. Even if they reach this discontinuity and are reflected, they are still largely absorbed by the critical level on their way back to the surface. This explains why the drag modulation is so small in Figs. $2 \mathrm{~b}$ and $3 \mathrm{~b}$ compared with Figs. $2 \mathrm{a}$ and $3 \mathrm{a}$. For a forward shear the decrease of the reflection coefficient from 1 at $\mathrm{Ri}=1 / 4$ to 0 as $\mathrm{Ri} \rightarrow+\infty$ is only due to smoothing of the shear discontinuity as Ri increases (as in Teixeira et al. 2005). This is the reason why the drag modulation in Figs. 2a or 3a increases as Ri decreases. For backward shear, on the other hand,

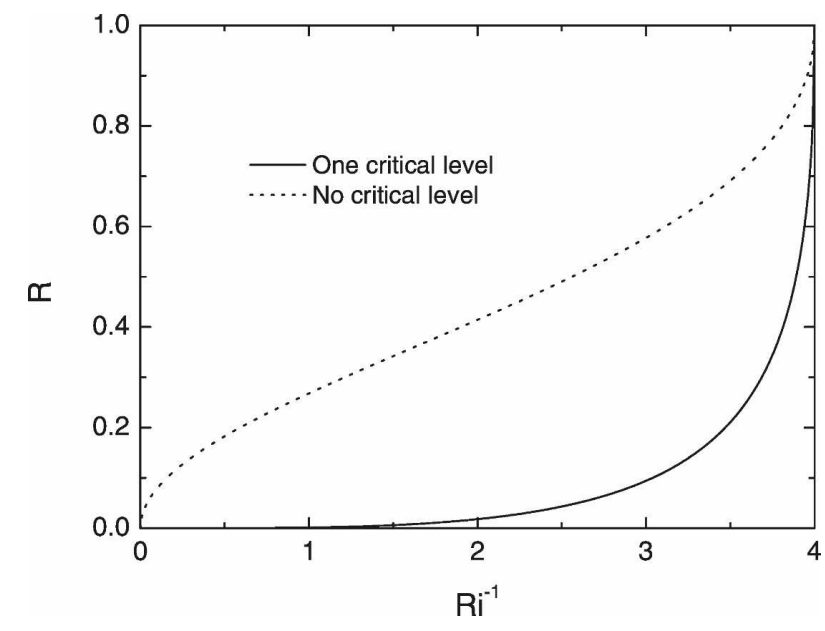

FIG. 4. Reflection coefficient of internal gravity waves as a function of $\mathrm{Ri}^{-1}$ for unidirectional shear flow over a $2 \mathrm{D}$ ridge.

this decay of the reflection coefficient to zero as $\mathrm{Ri}$ increases is much faster, because it is aided by the exponential factor in (23) (see Fig. 4). This explains the less erratic behavior of the drag in Fig. $2 b$, and its closeness to theories assuming an infinite shear layer.

There are two additional questions that need to be clarified. First, if the reflection coefficient is 1 for $\mathrm{Ri}=$ $1 / 4$ [the exponential factor in (23) is in this case irrelevant, being 1 itself], why does the drag tend to a finite value in this case, both with or without a critical level? There are two competing physical mechanisms at work here. On the one hand, in a hydrostatic framework, a reflection coefficient of 1 would tend to lead to an infinite number of upward and downward reflections of the waves, producing infinite drag in the case of constructive interference (see Teixeira et al. 2005). On the other hand, however, it is known from Grubišić and Smolarkiewicz (1997) and Teixeira and Miranda (2004) that constant-shear flows are characterized by a migration of the upstream pressure perturbation to the top of the mountain, with the pressure perturbation ultimately becoming symmetric with respect to the orography at $\mathrm{Ri}=1 / 4$. This is the physical reason for the prediction of zero drag at this Ri by exact linear theory (18) in the case of a shear that extends indefinitely. It can be shown that this behavior of the pressure also occurs in the present case. The two opposing effects described above balance each other, and explain the finite value of the drag at $\mathrm{Ri}=1 / 4$.

Another intriguing aspect is the following: since in flows both with and without a critical level $R=1$ at $\mathrm{Ri}=1 / 4$, why is the drag for this value of $\mathrm{Ri}$ smaller in the first case than in the second? This must be due to the fact that a critical level not only absorbs wave energy for $\mathrm{Ri}>1 / 4$, but also changes the wave phase, an 
effect that does not depend on $\mathrm{Ri}$ and so that also exists for $\mathrm{Ri}=1 / 4$. Therefore, passage of upward- and downward-propagating waves through the critical level when $\mathrm{Ri}=1 / 4$ presumably changes their phase in such a way as to partially counteract the effect of constructive interference, responsible for drag enhancement.

\section{b. Directional shear flow over a circular mountain}

After understanding the basic physical processes at work in 2D flows where reflection at a shear discontinuity and absorption at a critical level occur, it becomes easier to interpret 3D flows, with unidirectional or directional shear. The case of unidirectional shear is essentially similar to the $2 \mathrm{D}$ case treated above, because critical levels, if they exist, affect all wavenumbers. The only difference is that dispersion now modifies the waves, slightly smoothing the drag behavior (not shown). For directional shear, when this shear is constant and exists in a single layer, as assumed in the present study, some of the wavenumbers have critical levels, while others do not (provided these wavenumbers exist in the orographic forcing).

Flow over a circular mountain will be considered because of the simplifications this affords. Polar coordinates may then be used to express both the incoming wind,

$$
U=\mathcal{U} \cos \psi, \quad V=\mathcal{U} \sin \psi,
$$

and the wavenumber vector,

$$
k=\kappa \cos \theta, \quad l=\kappa \sin \theta,
$$

where $\mathcal{U} \geq 0$ and $\kappa \geq 0$ are the magnitudes of the wind velocity and of the horizontal wavenumber, respectively, and $\psi$ and $\theta$ are the corresponding azimuthal angles. This approach has the following advantages: First, the normalized drag is independent of the detailed shape of the orography, as for 2D flows (cf. Teixeira et al. 2004, 2005). This results from the possibility of separating the drag integral [(9)] into a part that depends only on $\kappa$ (which involves the orography shape) and a part that depends only on $\theta$. Second, a circular orography contains wavenumbers with all possible azimuthal angles, a feature that is convenient for illustrative purposes. Finally, in this coordinate system, a critical level in directional shear flow is expressed very simply as the level where $\cos (\theta-\psi)=0$, that is, where $\theta$ and $\psi$ are perpendicular to each other.

Figure 5 schematically shows (as the vertical hatching) the azimuthal angles spanned by the wind profiles with directional shear that are going to be considered in the following subsections, along with the azimuthal angles spanned by the wavenumbers that have critical levels (horizontal hatching). A wind profile similar to that shown in Fig. 5a, but where the shear extends indefinitely, was employed by Shutts (1995) and Shutts and Gadian (1999). The other two wind profiles, shown in Figs. 5b,c, are versions for a limited shear layer and for backward and forward shear, respectively, of one of the flows used by Teixeira et al. (2004).

The drag can be calculated using the appropriate expression (9) and normalized by its value for a constant wind equal to $\left(U_{0}, V_{0}\right)$, that is,

$$
\left(D_{0 x}, D_{0 y}\right)=4 \pi^{3} \rho_{0} N\left(U_{0}, V_{0}\right) \int_{0}^{+\infty} \kappa^{2}|\hat{h}|^{2} d \kappa
$$

(cf. Phillips 1984; Teixeira and Miranda 2006). The normalized drag is given by $1 \mathrm{D}$ integrals over $\theta$ and comprises four terms: two where the azimuthal angle $\theta$ spans the directions where the waves have not passed through a critical level (the regions without horizontal hatching in Fig. 5), and two other terms where $\theta$ spans the directions where the waves have passed through their critical level (the horizontally hatched regions in Fig. 5). In the first case, (12) must be used in the drag integrals, while in the second (14) must be used. Taking into account the symmetry of both the angles represented in Fig. 5 and the integrands, the normalized drag is in fact given by only two terms instead of four. When the wind turns anticlockwise, the corresponding expressions are

$$
\begin{aligned}
& \frac{D_{x}}{D_{0 x}}=\frac{2}{\pi}\left(\int_{\psi_{1}-\pi / 2}^{\psi_{0}+\pi / 2} I_{1} I_{3} d \theta+\int_{\psi_{0}+\pi / 2}^{\psi_{1}+\pi / 2} I_{1} I_{4} d \theta\right) \text { and } \\
& \frac{D_{y}}{D_{0 y}}=\frac{2}{\pi}\left(\int_{\psi_{1}-\pi / 2}^{\psi_{0}+\pi / 2} I_{2} I_{3} d \theta+\int_{\psi_{0}+\pi / 2}^{\psi_{1}+\pi / 2} I_{2} I_{4} d \theta\right),
\end{aligned}
$$

where

$$
\begin{aligned}
& I_{1}=\cos \theta\left(\cos \theta+\frac{V_{0}}{U_{0}} \sin \theta\right), \quad I_{2}=\sin \theta\left(\frac{U_{0}}{V_{0}} \cos \theta+\sin \theta\right), \quad I_{3}=\frac{\mu^{2} / \mathrm{Ri}_{\theta}}{1-\frac{1}{4 \mathrm{Ri}_{\theta}} \cos (2 \mu \lambda)-\frac{\mu}{2 \mathrm{Ri}_{\theta}} \sin (2 \mu \lambda)}, \quad \text { and } \\
& I_{4}=\frac{\frac{\mu^{2}}{\mathrm{Ri}_{\theta}} \cosh (2 \pi \mu)+\frac{\mu}{\mathrm{Ri}_{\theta}^{1 / 2}} \sinh (2 \pi \mu)}{\cosh (2 \pi \mu)+\frac{\mu}{\mathrm{Ri}_{\theta}^{1 / 2}} \sinh (2 \pi \mu)-\frac{1}{4 \mathrm{Ri}_{\theta}} \cos (2 \mu \lambda)-\frac{\mu}{2 \mathrm{Ri}_{\theta}} \sin (2 \mu \lambda)},
\end{aligned}
$$



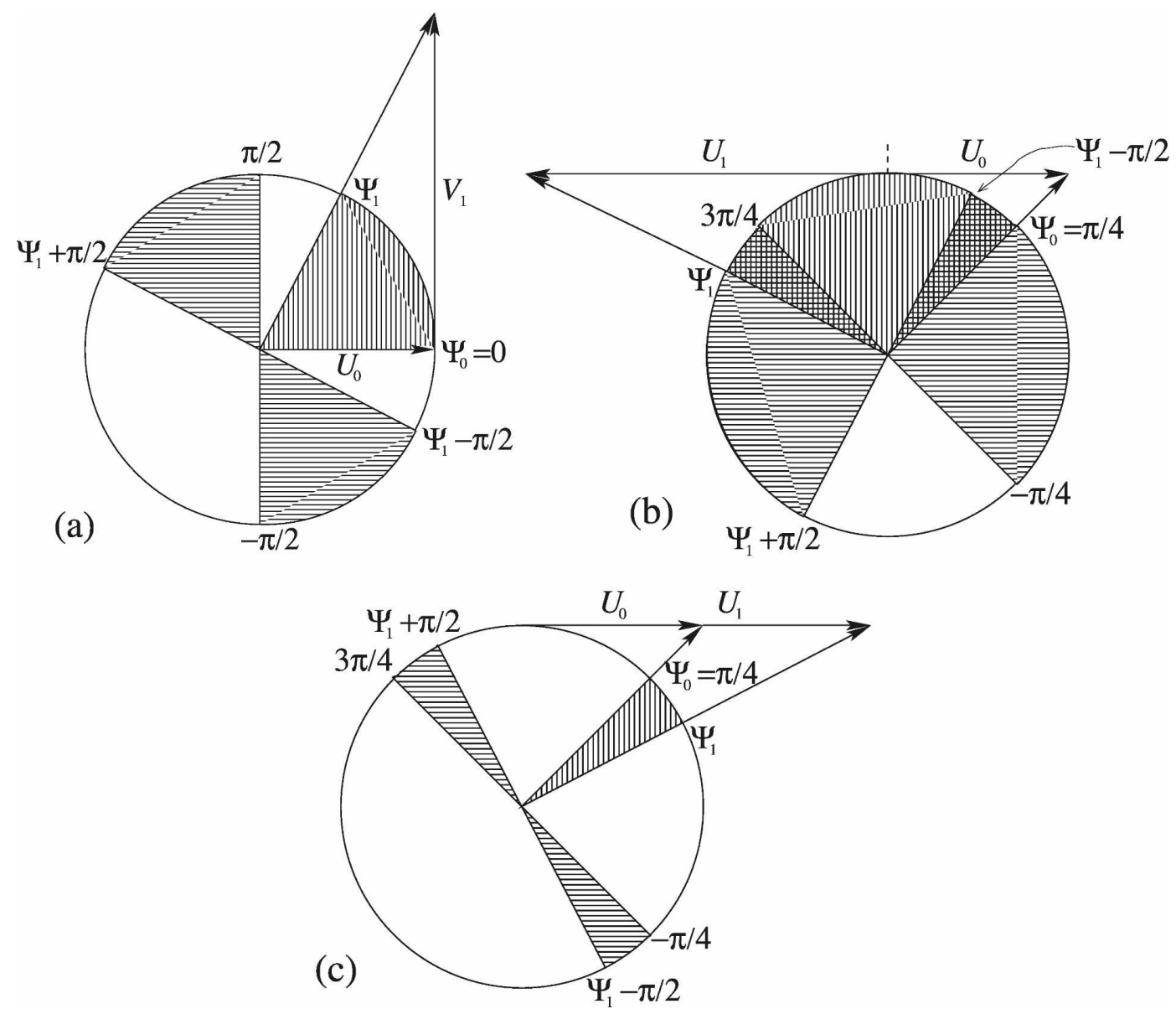

FIG. 5. Schematic diagram of the wind profiles with directional shear considered in this study. The azimuthal angles spanned by the wind profile (between $\psi_{0}$ and $\psi_{1}$ ) are denoted by the vertical hatching. The angles spanned by the wavenumbers filtered by the critical levels existing below $z=z_{1}$ are denoted by the horizontal hatching. (a) Wind profile of Shutts (1995), (b) wind profile of Teixeira et al. (2004) with backward shear, and (c) wind profile of Teixeira et al. (2004) with forward shear are described.

and

$$
\begin{aligned}
\lambda & =\log \left|\frac{U_{1} \cos \theta+V_{1} \sin \theta}{U_{0} \cos \theta+V_{0} \sin \theta}\right|, \quad \mu=\left(\mathrm{Ri}_{\theta}-\frac{1}{4}\right)^{1 / 2}, \quad \text { and } \\
\mathrm{Ri}_{\theta} & =\frac{N^{2}}{(\alpha \cos \theta+\beta \sin \theta)^{2}} .
\end{aligned}
$$

If the wind turns clockwise, $\psi_{0}$ and $\psi_{1}$ should be swapped in (27). The above equations can also be used in the case of unidirectional shear flow over a circular mountain, if it is noted that $\psi_{1}=\psi_{0}$ for flow without a critical level and $\psi_{1}=\psi_{0}+\pi$ for flow with a critical level. In (27), each component of the drag is normalized by the same component given by (26). This normalization is used so that the drag tends to 1 whenever possible, as $\mathrm{Ri} \rightarrow \infty$, but of course becomes invalid if either
$D_{0 x}$ or $D_{0 y}$ are zero. Then an alternative normalization, chosen on a case-by-case basis, must be adopted.

\section{1) Wind PRofile of Shutts (1995)}

Consider the wind profile (2), with $U_{0}>0, \alpha=0$, $V_{0}=0, \beta>0$, and, consequently, $U_{1}=U_{0}$ and $V_{1}>0$ (corresponding to Fig. 5a). The angles denoted in Fig. $5 \mathrm{a}$ and necessary in the drag integrals of (27) take the values $\psi_{0}=0$ and $\psi_{1}=\arctan \left(V_{1} / U_{0}\right)$. The normalized drag takes the form given in (27) together with (28) with the appropriate simplifications, and with the difference that the $y$ drag component must be normalized by $D_{0 x}$, because $D_{0 y}=0$. This corresponds to a simple multiplication of $D_{y} / D_{0 y}$ by the factor $V_{0} / U_{0}$ to yield $D_{y} / D_{0 x}$. For this flow, the normalized drag is a function of $\mathrm{Ri}=N^{2} / \beta^{2}$ and $\left|V_{1} / U_{0}\right|$. For a similar flow, but where the shear extends indefinitely, exact linear 

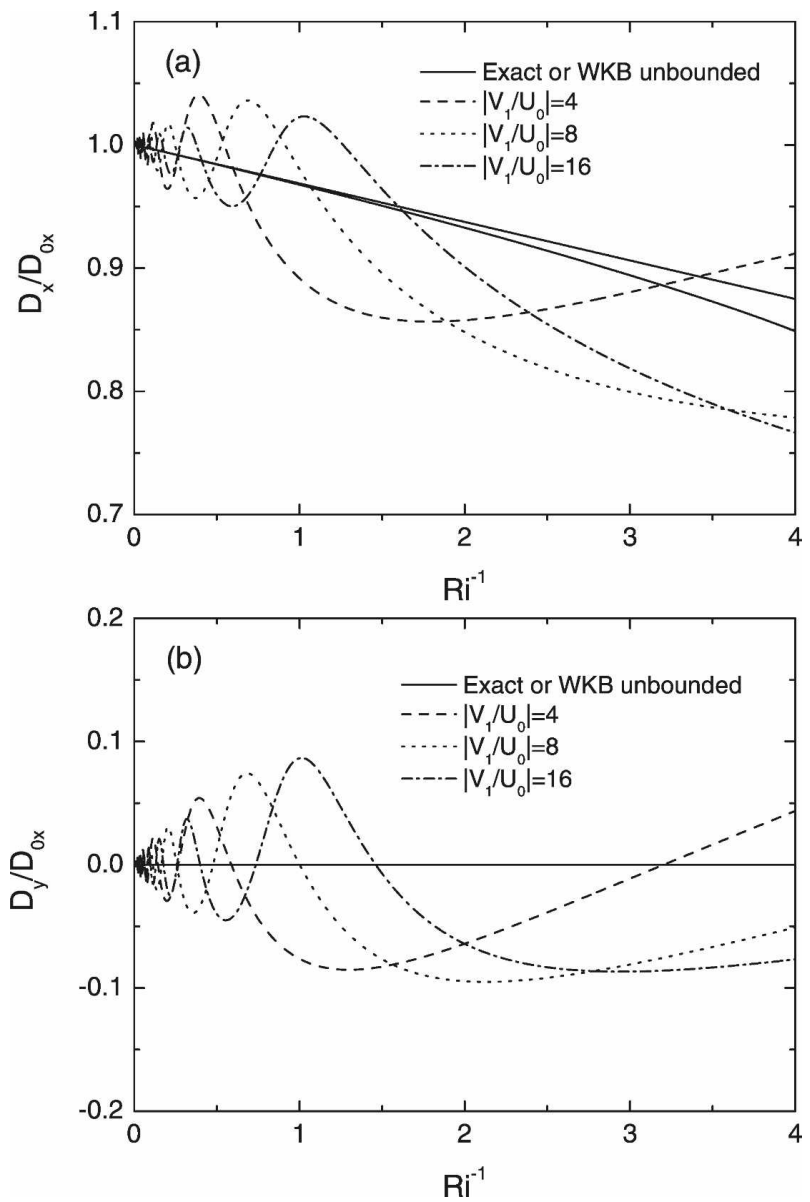

FIG. 6. Normalized drag as a function of $\mathrm{Ri}^{-1}$ for directional shear flow over a circular mountain, for the wind profile of Fig. 5a: (a) drag along $x$, (b) drag along $y$.

theory (Teixeira and Miranda 2006) predicts the normalized drag to be

$$
\begin{aligned}
\frac{D_{x}}{D_{0 x}} & =\frac{1}{\pi} \int_{0}^{2 \pi} \cos ^{2} \theta\left(1-\frac{1}{4 \mathrm{Ri}} \sin ^{2} \theta\right)^{1 / 2} d \theta \text { and } \\
\frac{D_{y}}{D_{0 x}} & =0
\end{aligned}
$$

while the theory based on the WKB approximation (cf. Teixeira et al. 2004) gives

$$
\frac{D_{x}}{D_{0 x}}=1-\frac{1}{32 \mathrm{Ri}} \text { and } \frac{D_{y}}{D_{0 x}}=0 .
$$

Note that both (30) and (31) do not depend on the sign of $\beta$.

Figure 6 shows (31) and (30) as the upper and lower solid lines in Fig. $6 \mathrm{a}$ and as the single solid line in Fig. $6 \mathrm{~b}$, as a function of Ri. The dashed, dotted, and dashdotted lines correspond to the limited shear-layer model developed in the present study for various values of $\left|V_{1} / U_{0}\right|$. A behavior of the drag that is quite similar to that shown previously in Fig. $2 \mathrm{a}$ is observed. The drag for a limited shear layer oscillates around the solid curves with a period and amplitude that increase as $\mathrm{Ri}$ decreases. This is due to wave reflection at the level $z=$ $z_{1}$, as in the previous flows. The physical reasons for this behavior are essentially the same as described in section 3a. However, now only a fraction of the wavenumbers (those with directions not spanned by the horizontal hatching in Fig. 5a) is reflected without further change. The remaining wavenumbers are filtered by the critical levels, so they presumably do not contribute to the drag oscillations, in accordance with the ideas expressed in section $3 \mathrm{~b}$. This is consistent with the somewhat lower relative modulation of the drag in Fig. 6 compared to Fig. 2a. On the other hand, the persistence of the oscillations has one curious consequence: the $y$ component of the drag becomes negative at relatively low Ri. This means that the drag has the opposite sign to the mean flow in the whole domain, and so the mountain tends to accelerate this component of the flow, unlike what is usual. This rather counterintuitive result, which was obtained first by the authors in numerical simulations, in fact partly motivated the present calculations. For symmetry reasons, if $\beta<0, D_{x}$ obviously takes the same values as calculated above for the same Ri and $\left|V_{1} / U_{0}\right|$, but $D_{y}$ takes symmetric values.

\section{2) Wind PROfile of TeIXeIRA ET AL. (2004)}

Consider now either the wind profile (2), with $U_{0}=$ $V_{0}>0, \alpha<0, U_{1}<0, \beta=0$, and $V_{1}=U_{0}$ (corresponding to Fig. $5 b$ ), or the same profile with the difference that $\alpha>0$ and consequently $U_{1}>0$ (Fig. 5c). The angles denoted in Figs. 5b,c take the values $\psi_{0}=$ $\pi / 4$ and $\psi_{1}=\arctan \left(U_{0} / U_{1}\right)$ in this case. The normalized drag is given by (27) together with (28) with the appropriate simplifications. Additionally, the drag given by exact linear theory for an infinite shear layer is

$$
\begin{aligned}
& \frac{D_{x}}{D_{0 x}}=\frac{1}{\pi} \int_{0}^{2 \pi} \cos ^{2} \theta\left(1-\frac{1}{4 \mathrm{Ri}} \cos ^{2} \theta\right)^{1 / 2} d \theta \text { and } \\
& \frac{D_{y}}{D_{0 y}}=\frac{1}{\pi} \int_{0}^{2 \pi} \sin ^{2} \theta\left(1-\frac{1}{4 \mathrm{Ri}} \cos ^{2} \theta\right)^{1 / 2} d \theta,
\end{aligned}
$$

where $\mathrm{Ri}=N^{2} / \alpha^{2}$, and the drag given by the theory based on the WKB approximation (Teixeira et al. 2004) is

$$
\frac{D_{x}}{D_{0 x}}=1-\frac{3}{32 \mathrm{Ri}} \text { and } \quad \frac{D_{y}}{D_{0 y}}=1-\frac{1}{32 \mathrm{Ri}}
$$



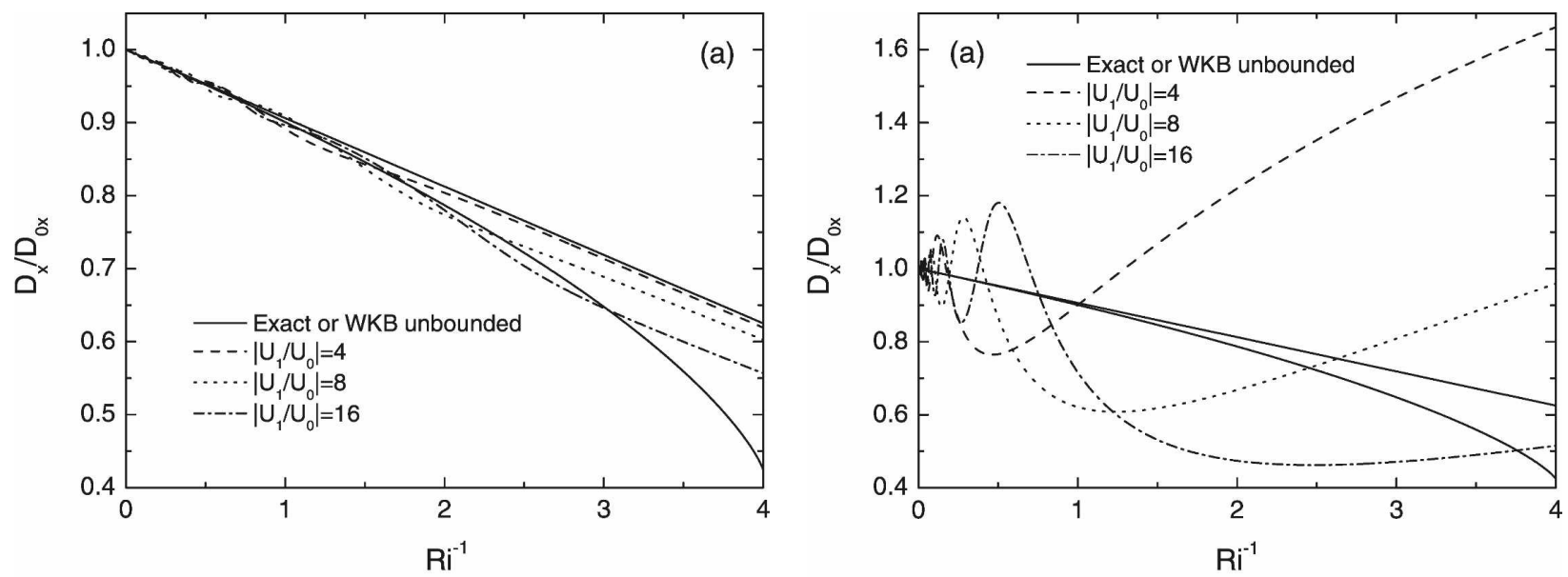

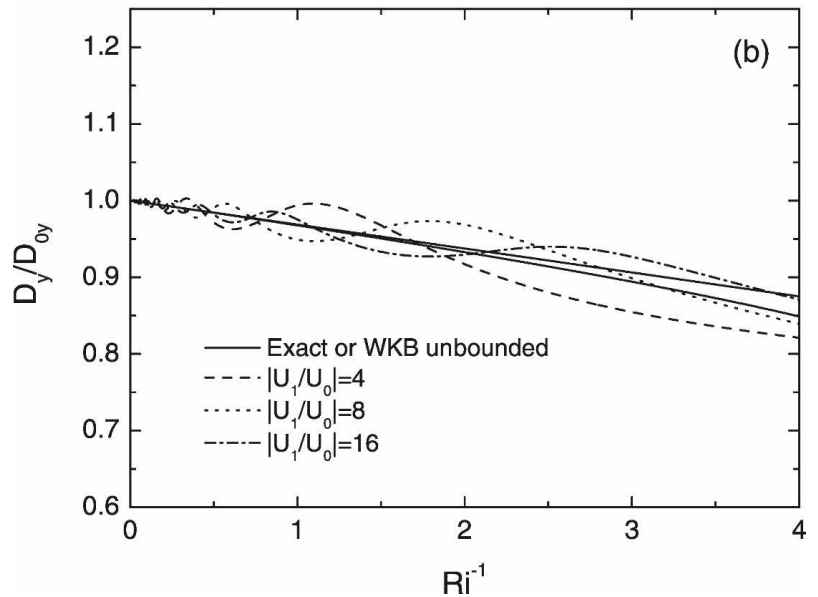

Fig. 7. Same as Fig. 6, but for wind profile of Fig. 5b.

It should again be noted that both (32) and (33) do not depend on the sign of $\alpha$.

Figure 7 shows the normalized drag as a function of $\mathrm{Ri}^{-1}$ for $\alpha<0$, and Fig. 8 shows the same for $\alpha>0$. In these figures, the upper solid curves correspond to (33) and the lower solid curves to (32). The dashed, dotted, and dash-dotted lines correspond to the model developed here for a finite shear layer for different values of $\left|U_{1} / U_{0}\right|$. The first observation suggested by these figures is that drag oscillations somewhat similar to those visible in Fig. 2a and Fig. 6 occur in Fig. 8, while they are virtually absent in Fig. 7a and are considerably reduced in Fig. 7b. These oscillations lead both components of the drag in Fig. 8 to take values much higher at low $\mathrm{Ri}$ than predicted by theories developed for an infinite shear layer. This is a consequence of the fact that the angle spanned by the wavenumbers filtered by a critical level is large in Fig. 5b, while it is small in Fig. 5c. This means that for $\alpha<0$ (backward shear of $U$ ) the majority of the wavenumbers are filtered by their critical levels in the same way as all of them are in Fig. 2b,

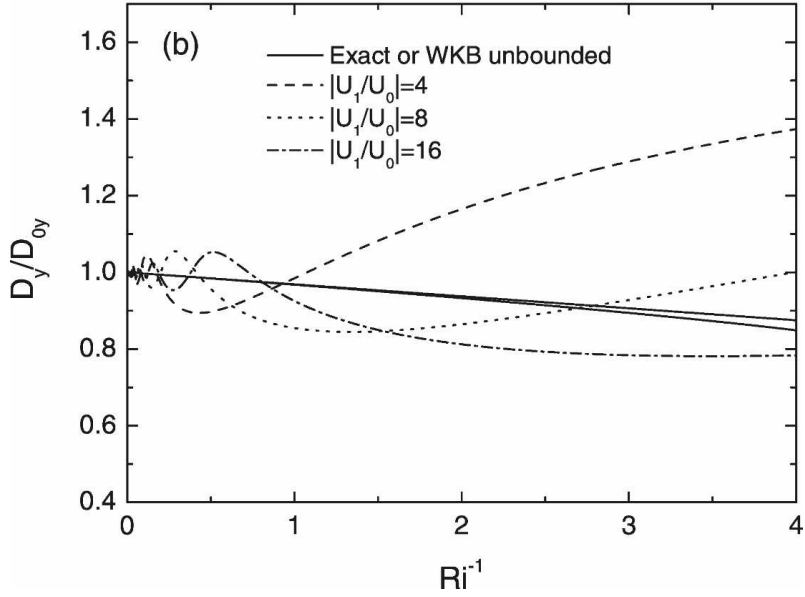

FIG. 8. Same as Fig. 6, but for wind profile of Fig. 5c.

while this only happens to a small fraction of the wavenumbers for $\alpha>0$ (forward shear). Therefore, in Fig. 7, the drag follows relatively closely the exact and WKB theories: in Fig. 7a, where $\left|U_{1} / U_{0}\right|=4$, for example, the drag is virtually coincident with the WKB prediction, and even for other values of $\left|U_{1} / U_{0}\right|$ stays essentially between the exact and the WKB results. The $y$ drag component behaves a little more erratically.

\section{c. Comparison with numerical results and nonlinear effects}

In this section, a few comparisons between the present analytical drag model and numerical simulations will be shown to exemplify its capabilities and limitations. Both linear conditions, where the model is expected to reproduce the numerical results, and weakly nonlinear conditions will be considered to see how the linear results are modified for more realistic (i.e., higher) mountains. The characteristics of the numerical runs carried out are described in the appendix. 

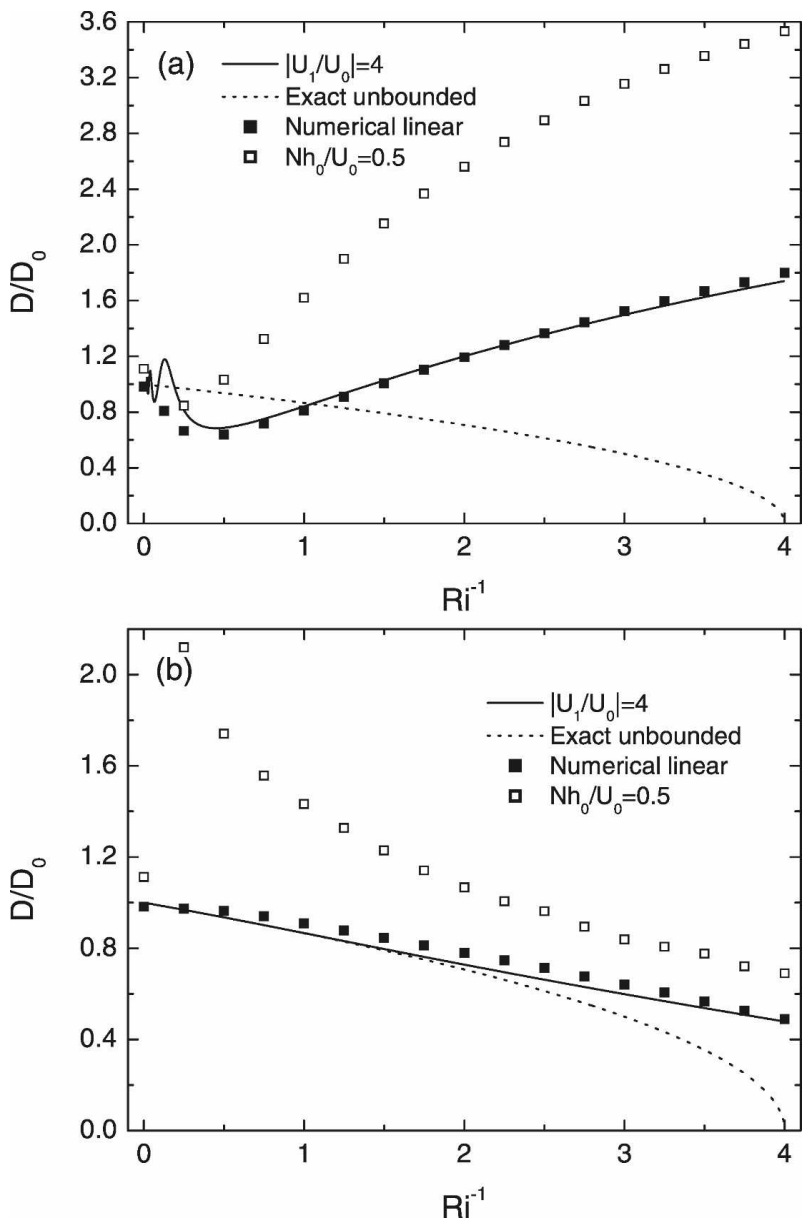

FIG. 9. Normalized drag as a function of $\mathrm{Ri}^{-1}$ for unidirectional shear flow over $2 \mathrm{D}$ ridge, for $\left|U_{1} / U_{0}\right|=4$ in linear and nonlinear conditions: (a) no critical level, (b) one critical level.

\section{1) $2 \mathrm{D}$ FLOWS}

Figure 9 shows the normalized drag for flow over a $2 \mathrm{D}$ ridge as a function of $\mathrm{Ri}^{-1}$. As can be seen, the model using a finite shear layer (denoted by the solid line) is able to capture the behavior of the numerical model in the linear regime (denoted by the solid squares) quite well. There are some discrepancies in Fig. 9a (for forward shear) at relatively low $\mathrm{Ri}^{-1}$, where the numerical model slightly underestimates the drag. This may be due to spurious damping of the momentum fluxes by the numerical scheme at high altitudes, a problem that was also verified in Teixeira et al. (2005), and is particularly acute here for large Ri because the shear discontinuity is then relatively high. The exact model for an infinite shear layer (denoted by the dotted line) is clearly inaccurate, because it fails to capture the differences between the case of forward and backward shear, as noted in earlier sections. The results from the numerical model in nonlinear conditions (denoted by the open squares) essentially show the same qualitative behavior as the linear results, but amplified by a considerable factor. In the presence of shear, the drag may be twice as large as in linear conditions. This is at variance with the relatively modest increase that the perturbation theory of Miles and Huppert (1969) predicts when $\mathrm{Ri}^{-1}=0$,

$$
\frac{D}{D_{0}}=1+\frac{7}{16}\left(\frac{N h_{0}}{U_{0}}\right)^{2}
$$

which for $N h_{0} / U_{0}=0.5$ gives $D / D_{0}=1.11$, essentially in agreement with Figs. 9a,b at $\mathrm{Ri}^{-1}=0$. There seems to be a qualitative change in behavior, with nonlinear effects becoming much more important, when $\mathrm{Ri}^{-1}=$ $O(1)$. This change is particularly marked and sudden (occurring between $\mathrm{Ri}^{-1}=0$ and $\mathrm{Ri}^{-1}=0.25$, and resembling a singularity) in the case of backward shear with a critical level (Fig. 9b). The explanation is probably related to nonlinear critical-level dynamics, as studied by Clark and Peltier (1984), and is beyond the scope of this study.

\section{2) $3 \mathrm{D}$ FLOWS}

In Figs. 10, 11, and 12, similar results for the flows with directional shear are presented. The symbols that were used have the same meaning as in Fig. 9. In Fig. 10, the flow used by Shutts (1995) is considered. As in flow over a ridge, the model developed in the present study is able to reproduce the results of the linear numerical simulations, but again these slightly underestimate the drag. The drag is considerably larger in nonlinear conditions, but the amplification is not as large as in Fig. 9. An interesting aspect is that the negative values of the $y$ component of the drag that are predicted in linear conditions remain valid in the nonlinear runs. Thus, the surface drag consistently opposes the corresponding velocity component of this wind profile at $\mathrm{Ri}^{-1}=1$, for example, contributing to a mean acceleration of the wind along $y$.

Figure 11 shows results for the wind profile of Teixeira et al. (2004) with backward shear. Again, the analytical model is able to predict the drag behavior of the numerical model in the linear regime satisfactorily. Although the $y$ component of the drag is almost in as good agreement with the analytical model (solid line) as it is with exact linear theory for an unlimited shear (dotted line), the drag maximum occurring around $\mathrm{Ri}^{-1}=1$ is well captured. The drag amplification in the nonlinear regime is smaller than in Fig. 9, but larger than in Fig. 10, with $D_{x}$ reaching a maximum near $\mathrm{Ri}^{-1}=0.5$ and $D_{y}$ near $\mathrm{Ri}^{-1}=0.75$. Because it was seen previously that the present wind profile has more critical levels than the preceding one, but less than a unidirectional flow, it is possible that the large drag 

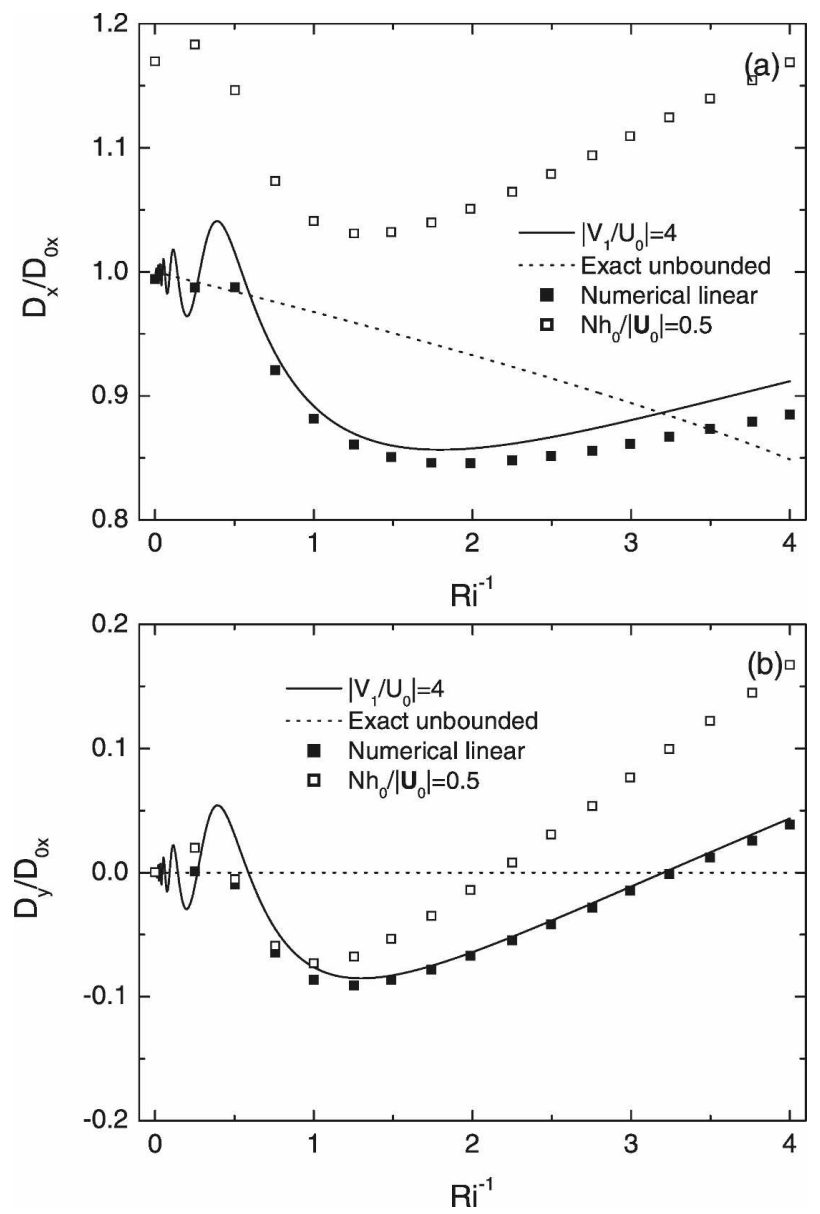

FIG. 10. Normalized drag as a function of $\mathrm{Ri}^{-1}$ for directional shear flow over a circular mountain, for the wind profile of Fig. 5a with $\left|V_{1} / U_{0}\right|=4$, in linear and nonlinear conditions: (a) drag along $x$, (b) drag along $y$.

amplification at relatively high $\mathrm{Ri}$ is due to nonlinear critical-level dynamics, as in Fig. 9b.

Finally, Fig. 12 displays results for the wind profile of Teixeira et al. (2004) with forward shear. The analytical model produces good predictions of the linear runs of the numerical model. Because of the small range of angles spanned by wavenumbers with a critical level, the two components of the drag essentially behave as in a situation of forward unidirectional shear, showing some considerable enhancement at $\mathrm{Ri}=O(1)$. However, the drag amplification at $N h_{0} /\left(U_{0}^{2}+V_{0}^{2}\right)^{1 / 2}=0.5$ is still somewhat reduced relative to the case of flow over a ridge (Fig. 2a), presumably because of the wave dispersion inherent to a 3D flow.

\section{Concluding remarks}

An essential finding of the present study, substantiated by the preceding results, is that internal wave drag
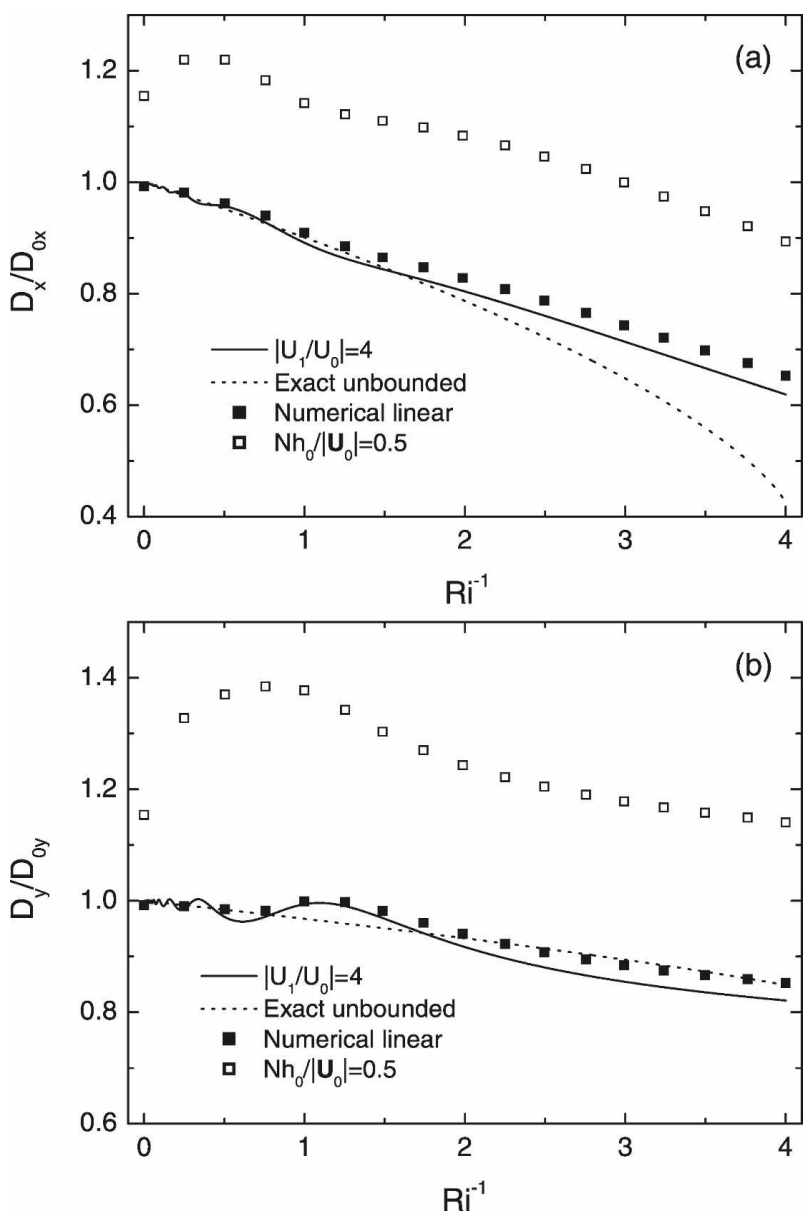

FIG. 11. Same as Fig. 10, but for the wind profile of Fig. 5b.

behaves totally differently for hydrostatic flows with and without critical levels when the shear is limited to a layer of finite extent near the surface. This result is relevant, because linear theory for an unlimited constant-shear layer predicts no differences between these two cases. However, in numerical models the simplest way to approximate a constant-shear flow is by making the wind velocity become constant above some prescribed height. The present results show that this may be a relatively good approximation for low mountains in the case of unidirectional shear flow with a critical level, but not in the case of a flow without critical levels (e.g., forward shear), where wave reflections at the shear discontinuity substantially modify the drag behavior. For the flow with a critical level, the drag is in surprisingly good agreement with corresponding results for a shear that extends indefinitely, particularly when the WKB approximation is used, despite the fact that critical levels become transparent to internal waves at low $\mathrm{Ri}$. This is due to filtering of the waves by these levels, which suppresses the oscillating drag behavior. 

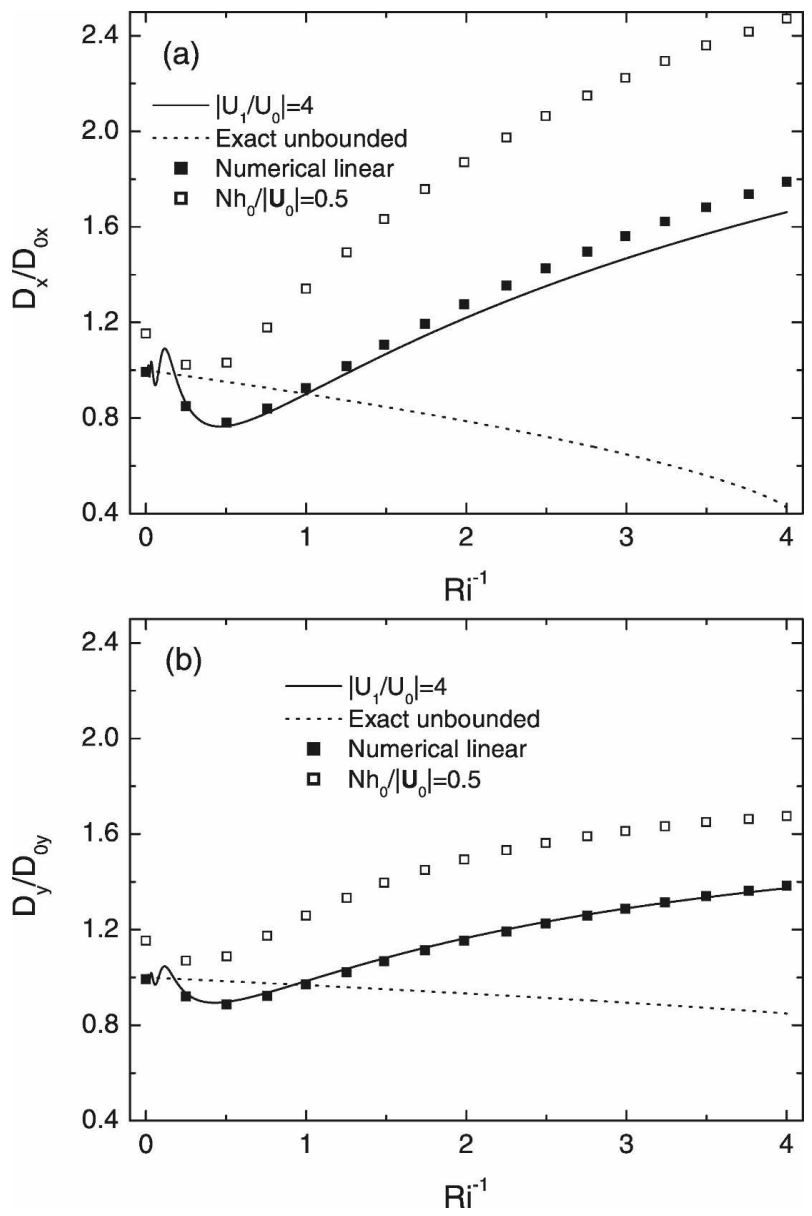

FIG. 12. Same as Fig. 10, but for the wind profile of Fig. 5c.

In directionally sheared flows, what appears to be crucial for determining the drag behavior is the fraction of wavenumbers that encounter their critical levels before they are reflected at the shear discontinuity. If this fraction is large, the waves are filtered in such a way that the reflection seems to be of only modest importance, while if this fraction is small, the reflection process completely determines the drag, generally enhancing it. These aspects were not contemplated in previous drag models, and clearly must be taken into account in certain situations of practical importance, when there are relatively fast variations of the shear with height. When the shear variations occur over sufficiently deep regions, or dissipative processes act before they are reached by the waves, the drag presumably is dominated by local mechanisms, as assumed in the WKB treatments of Teixeira et al. (2004) or Teixeira and Miranda (2006).

From a fundamental point of view, the present study contributes to the improvement of our knowledge about physical processes occurring above critical levels, a topic about which not much has been said yet, because of the frequent assumption of high $\mathrm{Ri}$ and the consequent total wave absorption (Shutts 1995; Shutts and Gadian 1999).

Nonlinear processes have been shown to modify the linear results by generally increasing the value of the drag. This increase is, however, considerably larger than that predicted by the theory of Miles and Huppert (1969). While in the range of $\mathrm{Ri}^{-1}$ considered in the present study (excepting $\mathrm{Ri}^{-1}=0$ ) the qualitative behavior of the drag with $\mathrm{Ri}^{-1}$ is essentially unchanged for $N h_{0} /\left(U_{0}^{2}+V_{0}^{2}\right)^{1 / 2}=0.5$, there appears to be an interaction of shear and nonlinear effects that potentiates the drag enhancement as long as $\mathrm{Ri}$ is not extremely large. This leads to an apparent singularity in the drag behavior for unidirectional flow with a critical level, which deserves further investigation. Grubišić and Smolarkiewicz (1997) suggested that nonlinear effects become progressively more important as $\mathrm{Ri}$ decreases, and might be responsible for the slightly worse agreement between linear theory and their numerical simulation results at low $\mathrm{Ri}$. The present findings explain similar differences, in the linear regime, simply by wave reflection [suggesting that Grubišić and Smolarkiewicz (1997) may have used a shear discontinuity above the critical level]. Our findings also suggest that when nonlinear processes become important, these processes are much stronger if there is any appreciable shear, not only at low Ri. This result has obvious relevance for real flows, because wind profiles completely devoid of shear must be very rare.

Acknowledgments. This work was supported by Fundação para a Ciência e Tecnologia (FCT) under Project BOSS, Contract POCI/CTE-ATM/58932/2004, cofinanced by the European Union under program FEDER.

\section{APPENDIX}

\section{Setup of the Numerical Runs}

The numerical simulations were carried out using two different models, developed independently. Flow over a ridge was simulated using the FLEX model and flow over a circular mountain used the NH3D model. FLEX (see Argaín 2003) is a 2D numerical model using curvilinear coordinates and allowing local grid refinement, which can be used from the microscale to the mesoscale. NH3D (see Miranda and James 1992) is a 3D mesoscale model using a pressure-based terrainfollowing vertical coordinate. Although both models 
are nonhydrostatic and parameterize turbulent processes, they were run in inviscid and approximately hydrostatic conditions.

The simulations of flow over a ridge used a domain of $160 \times 250$ grid points, with uniform horizontal and vertical spacings of 1600 and $80 \mathrm{~m}$, respectively. The time step was $10 \mathrm{~s}$ and the model was run for 6400 time steps until the drag stabilized to a constant value. A bellshaped ridge with height $h_{0}=10 \mathrm{~m}$ and half-width $a=$ $16 \mathrm{~km}$ was considered in the linear runs, while $h_{0}=500$ $\mathrm{m}$ was used in the nonlinear runs. The wind speed at the surface was $U_{0}=10 \mathrm{~m} \mathrm{~s}^{-1}$ and a constant BruntVäisälä frequency of $N=0.01 \mathrm{~s}^{-1}$ was employed. This gives $N a / U_{0}=16$, indicating that the flow was mostly hydrostatic. Additionally, $N h_{0} / U_{0}=0.01$ at the surface for the linear runs and $N h_{0} / U_{0}=0.5$ for the nonlinear runs. A sponge was applied at the lateral boundaries over a distance $2 a=32 \mathrm{~km}$ and at the top of the domain over a distance $3 \pi U_{0} / N=9425 \mathrm{~m}$.

In the simulations of flow over a (bell shaped) circular mountain, the domain was of $100 \times 100 \times 200$ grid points, with spacings of 2000 or $3000 \mathrm{~m}$ in the horizontal (respectively, when the wind was aligned or oblique to the grid at the surface) and between 40 (near the surface) and $400 \mathrm{~m}$ in the vertical. The number of time steps was 10000 and the time step was 4 or $6 \mathrm{~s}$ for the two cases mentioned above. Sponges spanning 10 grid points (20 or $30 \mathrm{~km}$, respectively) were used at the lateral boundaries and a sponge above $z=10 \mathrm{~km}$ was applied at the top of the domain. The mountain height was $h_{0}=5 \mathrm{~m}$ or $h_{0}=7.07 \mathrm{~m}$ in the linear runs and $h_{0}=250 \mathrm{~m}$ or $h_{0}=353 \mathrm{~m}$ in the nonlinear runs, and the half-width of the mountain was $a=10 \mathrm{~km}$ or $a=14.14$ $\mathrm{km}$. Finally, $N=0.01 \mathrm{~s}^{-1}$ and $\left(U_{0}^{2}+V_{0}^{2}\right)^{1 / 2}=5 \mathrm{~m} \mathrm{~s}^{-1}$ or $\left(U_{0}^{2}+V_{0}^{2}\right)^{1 / 2}=7.07 \mathrm{~m} \mathrm{~s}^{-1}$. This gives $\mathrm{Na} /\left(U_{0}^{2}+\right.$ $\left.V_{0}^{2}\right)^{1 / 2}=20$ in all simulations, again ensuring nearly hydrostatic conditions, and $N h_{0} /\left(U_{0}^{2}+V_{0}^{2}\right)^{1 / 2}=0.01$ or $N h_{0} /\left(U_{0}^{2}+V_{0}^{2}\right)^{1 / 2}=0.5$, respectively, for the linear and nonlinear runs.

\section{REFERENCES}

Argaín, J. L., 2003: Numerical modelling of atmospheric flow: Orographic and boundary layer effects. Ph.D. thesis, University of Algarve, $249 \mathrm{pp}$.

Booker, J. R., and F. P. Bretherton, 1967: The critical layer for internal gravity waves in a shear flow. J. Fluid Mech., 27, 513-539.

Clark, T. L., and W. R. Peltier, 1984: Critical level reflection and the resonant growth of nonlinear mountain waves. J. Atmos. Sci., 41, 3122-3134.

Grubišić, V., and P. K. Smolarkiewicz, 1997: The effect of critical levels on 3D orographic flows: Linear regime. J. Atmos. Sci., 54, 1943-1960.
Keller, T. L., 1994: Implications of the hydrostatic assumption on atmospheric gravity waves. J. Atmos. Sci., 51, 1915-1929.

Klemp, J. B., and D. R. Lilly, 1975: The dynamics of wave-induced downslope winds. J. Atmos. Sci., 32, 320-339.

Lindzen, R. S., and K.-K. Tung, 1976: Banded convective activity and ducted gravity waves. Mon. Wea. Rev., 104, 1602-1617.

Miles, J. W., and H. E. Huppert, 1969: Lee waves in a stratified flow. Part 4. Perturbation approximation. J. Fluid Mech., 35, 497-525.

Miranda, P. M. A., and I. N. James, 1992: Non-linear threedimensional effects on the wave drag: Splitting flow and breaking waves. Quart. J. Roy. Meteor. Soc., 118, 1057-1081.

, and M. A. Valente, 1997: Critical level resonance in threedimensional flow past isolated mountains. J. Atmos. Sci., 54, 1574-1588.

Öllers, M. C., L. P. J. Kamp, F. Lott, P. F. J. van Velthoven, H. M. Kelder, and F. W. Sluijter, 2003: Propagation properties of inertia-gravity waves through a barotropic shear layer and application to the Antarctic polar vortex. Quart. J. Roy. Meteor. Soc., 129, 2495-2511.

Phillips, D. S., 1984: Analytical surface pressure and drag for linear hydrostatic flow over three-dimensional elliptical mountains. J. Atmos. Sci., 41, 1073-1084.

Sharman, R. D., and M. G. Wurtele, 2004: Three-dimensional structure of forced gravity waves and lee waves. J. Atmos. Sci., 61, 664-681.

Shen, B.-W., and Y.-L. Lin, 1999: Effects of critical levels on two-dimensional back-sheared flow over an isolated mountain ridge on an $f$ plane. J. Atmos. Sci., 56, 3286-3302.

Shutts, G., 1995: Gravity-wave drag parameterization over complex terrain: The effect of critical-level absorption in directional wind-shear. Quart. J. Roy. Meteor. Soc., 121, 10051021.

2001: A linear model of back-sheared flow over an isolated hill in the presence of rotation. J. Atmos. Sci., 58, 3293-3311. -, and A. Gadian, 1999: Numerical simulations of orographic gravity waves in flows which back with height. Quart. J. Roy. Meteor. Soc., 125, 2743-2765.

Smith, R. B., 1984: A theory of lee cyclogenesis. J. Atmos. Sci., 41, 1159-1168.

- 1986: Further development of a theory of lee cyclogenesis. $J$. Atmos. Sci., 43, 1582-1602.

Teixeira, M. A. C., and P. M. A. Miranda, 2004: The effect of wind shear and curvature on the gravity wave drag produced by a ridge. J. Atmos. Sci., 61, 2638-2643.

, and - 2005: Linear criteria for gravity-wave breaking in resonant stratified flow over a ridge. Quart. J. Roy. Meteor. Soc., 131, 1815-1820.

$\longrightarrow$, and - 2006: A linear model of gravity wave drag for hydrostatic sheared flow over elliptical mountains. Quart. J. Roy. Meteor. Soc., 132, 2439-2458.

- - $\longrightarrow$, and M. A. Valente, 2004: An analytical model of mountain wave drag for wind profiles with shear and curvature. J. Atmos. Sci., 61, 1040-1054.

,,- J. L. Argaín, and M. A. Valente, 2005: Resonant gravity-wave drag enhancement in linear stratified flow over mountains. Quart. J. Roy. Meteor. Soc., 131, 1795-1814.

Wang, T.-A., and Y.-L. Lin, 1999: Wave ducting in a stratified shear flow over two-dimensional mountain. Part I: General linear criteria. J. Atmos. Sci., 56, 412-436.

Wurtele, M. G., A. Datta, and R. D. Sharman, 2000: The propagation of a gravity-inertia wave in a positively sheared flow. J. Atmos. Sci., 57, 3703-3715. 